BULL. AUSTRAL. MATH. SOC.

VOL. $12(1975), 9-21$.

\title{
On the absolute Nörlund summability factors of Fourier series
}

\section{Yasuo Okuyama}

The object of this paper is to give a general theorem which implies Izumi's Theorem and Kanno's Theorem on the absolute Nörlund sumability factors of Fourier series and deduce to several known and new results from the theorem.

\section{1.}

Let $\sum a_{n}$ be a given infinite series with the sequence of partial sums $\left\{s_{n}\right\}$. Let $\left\{p_{n}\right\}$ be a sequence of constants, real or complex, and let us write

$$
P_{n}=p_{0}+p_{1}+\ldots+p_{n} ; P_{-k}=p_{-k}=0 \text {, for } k \geq 1 .
$$

The sequence $\left\{t_{n}\right\}$, given by

$$
t_{n}=\frac{1}{P_{n}} \sum_{k=0}^{n} p_{n-k} s_{k}=\frac{1}{P_{n}} \sum_{k=0}^{n} P_{k} a_{n-k}, \quad\left(P_{n} \neq 0\right),
$$

defines the Nörlund means of the sequence $\left\{s_{n}\right\}$ generated by the sequence of constants $\left\{p_{n}\right\}$.

The series $\sum a_{n}$ is said to be absolutely summable $\left(N, p_{n}\right)$, or summable $\left|N, p_{n}\right|$, if the series

$$
\sum_{n=1}^{\infty}\left|t_{n}-t_{n-1}\right|
$$

Received 11 September 1974. Communicated by Shin-ichi Izumi. 
is convergent.

In the special cases in which $p_{n}=\Gamma(n+\alpha) / \Gamma(\alpha) \Gamma(n+1), \alpha>0$, and $p_{n}=1 /(n+1)$, summability $\left|N, p_{n}\right|$ are the same as the summability $|c, \alpha|$ and the absolute harmonic summability, respectively.

\section{2 .}

Let $f(t)$ be a periodic function with period $2 \pi$ and integrable $(L)$ over $(-\pi, \pi)$. We assume without any loss of generality that the Fourier series of $f(t)$ is given by

$$
\sum_{n=1}^{\infty}\left(a_{n} \cos n t+b_{n} \sin n t\right)=\sum_{n=1}^{\infty} A_{n}(t)
$$

and $\int_{-\pi}^{\pi} f(t) d t=0$. We write $\varphi_{x}(t)=\varphi(t)=\frac{1}{2}\{f(x+t)+f(x-t)\}$, $\lambda(n)=\lambda_{n}$, and $\Delta \lambda_{n}=\lambda_{n}-\lambda_{n+1}$.

Dealing with the absolute Nörlund summability of Fourier series, Izumi and Izumi [3] proved the following theorem, which is a generalization of theorems due to Bosanquet [I] and Mohanty [6, 7].

THEOREM A. Let $\left\{p_{n}\right\}$ be non-negative and non-increasing and $\lambda(t)$, $t>0$, be a positive non-decreasing function such that $\left\{\lambda_{n} /(n+1)\right\}$ is non-increasing,

$$
\sum_{k=n}^{\infty} \frac{\lambda_{k}}{(k+1) P_{k}}=0\left(\frac{\lambda_{n}}{P_{n}}\right), n=0,1,2, \ldots
$$

and

$$
\int_{0}^{\pi} \lambda(c / t)|d \varphi(t)|<\infty .
$$

Then the series

$$
\sum_{n=0}^{\infty} \lambda_{n} A_{n}(t)
$$

is sumable $\left|N, p_{n}\right|$, at $t=x$. 
Generalizing the theorem of Varshney [8], Kanno [4] proved the following theorem.

THEOREM B. Let $\left\{p_{n}\right\}$ be non-negative and non-increasing. Let $\lambda(t), t>0$, be a positive, non-decreasing function satisfying the condition $\left\{\lambda_{n} / P_{n}\right\}$ is non-increasing.

If the conditions

$$
\sum_{k=n}^{\infty} \frac{p_{k} \lambda_{k}}{P_{k}^{2}}=0\left(\frac{\lambda_{n}}{P_{n}}\right), n=0,1,2, \ldots
$$

and

$$
\int_{0}^{\pi} \lambda(c / t)|d \varphi(t)|<\infty
$$

for some constant $C>0$ hold, then the series

$$
\sum_{n=0}^{\infty} \frac{(n+1) p_{n}}{P_{n}} \lambda_{n} A_{n+1}(t)
$$

is summable $\left|N, p_{n}\right|$ at $t=x$.

Also see Dikshit [2] for the proofs of these theorems.

We generalize these theorems in the following form.

THEOREM. Let $\left\{p_{n}\right\}$ be non-negative and non-increasing. Suppose that $\left\{\lambda_{n}^{(1)}\right\}$ is a positive bounded sequence and $\lambda^{(2)}(t), t>0$, is a positive non-decreasing function such that $\left\{\lambda_{n}^{(1)} \lambda_{n}^{(2)} /(n+1)\right\}$ is nonincreasing,

$$
\sum_{k=n}^{\infty} \frac{\lambda_{k}^{(1)} \lambda_{k}^{(2)}}{k P_{k}}=0\left(\frac{\lambda_{n}^{(2)}}{P_{n}}\right), n=1,2, \ldots
$$

and

$$
\int_{0}^{\pi} \lambda^{(2)}(C / t)|d \varphi(t)|<\infty \text {, for a constant } C(>2 \pi) \text {. }
$$

Then the series 


$$
\sum_{n=0}^{\infty} \lambda_{n}^{(1)} \lambda_{n}^{(2)} A_{n+1}(t)
$$

is sumable $\left|N, p_{n}\right|$, at $t=x$.

If $\lambda_{n}^{(1)}=1$ and $\lambda_{n}^{(2)}=\lambda_{n}$, our theorem reduces to Theorem A. If we put $\lambda_{n}^{(1)}=(n+1) p_{n} / P_{n}$ and $\lambda_{n}^{(2)}=\lambda_{n}$, we easily see that, under the same assumptions as those of Theorem B, the condition (2.6) is satisfied and the sequence $\left\{\lambda_{n}^{(1)} \lambda_{n}^{(2)} /(n+1)\right\}$ is non-increasing because

$$
\sum_{k=n}^{\infty} \frac{\lambda_{n}^{(1)} \lambda_{n}^{(2)}}{k P_{k}} \leq A \sum_{k=n}^{\infty} \frac{p_{k} \lambda_{k}^{(2)}}{P_{k}^{2}}=o\left(\frac{\lambda_{n}^{(2)}}{P_{n}}\right)
$$

and

$$
\frac{\lambda_{n}^{(1)} \lambda_{n}^{(2)}}{n+1}=\frac{p_{n} \lambda_{n}^{(2)}}{P_{n}}
$$

Therefore our theorem includes Theorem B.

3.

We shall require the following lemmas to prove the theorem.

LEMMA 1 [2]. Let $\left\{p_{n}\right\}$ be a given sequence, then for any $x$, we have

$$
\text { (1-x) } \sum_{k=m}^{n} p_{k} x^{k}=p_{m}^{x^{m}}-p_{n} x^{n+1}-\sum_{k=m}^{n-1} \Delta p_{k} x^{k+1}
$$

where $m$ and $n$ are integers such that $n \geq m \geq 0$.

This lemma is easily obtained.

LEMMA 2 [5]. If $\left\{p_{n}\right\}$ is non-negative, non-increasing, then for $0 \leq a \leq b<\infty, 0 \leq t \leq \pi$, and for any $n$; we have

$$
\left|\sum_{k=\alpha}^{b} p_{k} \exp (i(n-k) t)\right| \leq A P_{[1 / t]} \text {. }
$$


where $A$ is a positive constant and $[x]$ denotes the integral part of $x$.

4.

Proof of the Theorem. By (1.1) we have

$$
t_{n}=\frac{1}{P_{n}} \sum_{k=0}^{n} P_{k} \lambda_{n-k}^{(1)} \lambda_{n-k}^{(2)} A_{n+1-k}(t),
$$

where

$$
A_{k}(x)=\frac{2}{\pi} \int_{0}^{\pi} \varphi(t) \cos k t d t
$$

Hence,

$$
\begin{aligned}
t_{n}-t_{n-1} & =\sum_{k=1}^{n}\left(\frac{P_{n-k}}{P_{n}}-\frac{P_{n-k-1}}{P_{n-1}}\right) \lambda_{k}^{(1)} \lambda_{k}^{(2)} A_{k+1}(t) \\
& =\frac{2}{\pi} \int_{0}^{\pi} \varphi(t)\left\{\frac{1}{P_{n} P_{n-1}} \sum_{k=1}^{n}\left(P_{n} p_{n-k}-P_{n-k} p_{n}\right) \lambda_{k}^{(1)} \lambda_{k}^{(2)} \cos (k+1) t\right\} d t \\
& =\frac{2}{\pi} \int_{0}^{\pi} d \varphi(t)\left\{\frac{1}{P_{n} P_{n-1}} \sum_{k=1}^{n}\left(P_{n} p_{n-k}-P_{n-k} p_{n}\right) \lambda_{k}^{(1)} \lambda_{k}^{(2)} \frac{\sin (k+1) t}{k+1}\right\} .
\end{aligned}
$$

Thus, to prove the theorem, it is enough to show that

$$
\begin{aligned}
& \sum_{n=1}^{\infty}\left|t_{n}-t_{n-1}\right| \\
& \quad \leq \frac{2}{\pi} \int_{0}^{\pi}|d \varphi(t)|\left|\sum_{n=1}^{\infty} \frac{1}{P_{n}^{P} n-1} \sum_{k=1}^{n}\left(P_{n} p_{n-k}-P_{n-k} p_{n}\right) \lambda_{k}^{(1)} \lambda_{k}^{(2)} \frac{\sin (k+1) t}{k+1}\right|=O(1) .
\end{aligned}
$$

Considering the condition (2.7), it suffices for our purpose to prove that uniformly in $0<t \leq \pi$,

$$
\text { (4.1) } I=\sum_{n=1}^{\infty}\left|\sum_{k=1}^{n} \frac{\left(P_{n} p_{n-k}-P_{n-k^{p}}\right)}{P_{n} P_{n-1}} \lambda_{k}^{(1)} \lambda_{k}^{(2)} \frac{\sin (k+1) t}{k+1}\right|=O\left(\lambda^{(2)}(C / t)\right) \text {. }
$$

Let us write $\tau=[C / 2 t]$, so we have 


$$
\begin{aligned}
I \leq \sum_{n=1}^{2 \tau+1}\left|\sum_{k=1}^{n} \frac{\left(P_{n} p_{n-k^{-P}} P_{n-k} p_{n}\right)}{P_{n} P_{n-1}} \lambda_{k}^{(1)} \lambda_{k}^{(2)} \frac{\sin (k+1) t}{k+1}\right| \\
\left.+\sum_{n=2 \tau+2}^{\infty}\left|\sum_{k=1}^{\tau}\right| \frac{n-k}{P_{n}}-\frac{P_{n-k-1}}{P_{n-1}}\right) \lambda_{k}^{(1)} \lambda_{k}^{(2)} \frac{\sin (k+1) t}{k+1} \mid \\
+\sum_{n=2 \tau+2}^{\infty}\left|\sum_{k=\tau+1}^{n}\left(\frac{P_{n-k}}{P_{n}}-\frac{P_{n-k-1}}{P_{n-1}}\right) \lambda_{k}^{(1)} \lambda_{k}^{(2)} \frac{\sin (k+1) t}{k+1}\right| \\
=I_{1}+I_{2}+I_{3},
\end{aligned}
$$

say.

Since $\left\{p_{n}\right\}$ is non-negative and non-increasing, $\left\{\lambda_{n}^{(1)}\right\}$ is bounded, $\left\{\lambda_{n}^{(2)}\right\}$ is non-decreasing and $|\sin (k+1) t| \leq(k+1) t$, we have

$$
\begin{aligned}
I_{1} \leq A t \sum_{n=1}^{2 \tau+1} \lambda_{n}^{(2)} \frac{1}{P_{n}} \sum_{k=1}^{n} p_{n-k} & \leq A \lambda^{(2)}(C / t) \cdot t \cdot 2 \tau \\
& =O\left(\lambda^{(2)}(C / t)\right) .
\end{aligned}
$$

Also, since $\left\{P_{n-k} / P_{n}\right\}$ is monotonic non-decreasing and bounded for each fixed $k \geq 0$, we have

$$
\begin{aligned}
I_{2} \leq A t \sum_{k=1}^{\tau} \lambda_{k}^{(2)} \sum_{n=2 \tau+2}^{\infty}\left(\frac{n-k}{P_{n}}-\frac{P_{n-k-1}}{P_{n-1}}\right) & \leq A t \lambda^{(2)}(C / t) \sum_{k=1}^{\tau} 1 \\
& =o\left(\lambda^{(2)}(c / t)\right),
\end{aligned}
$$

by virtue of the hypotheses that $\left\{\lambda_{n}^{(1)}\right\}$ is bounded and $\left\{\lambda_{n}^{(2)}\right\}$ is nondecreasing.

In order to prove that $I_{3}=O\left(\lambda^{(2)}(c / t)\right)$, we consider the sum

$$
I_{3}^{*}=\sum_{n=2 \tau+2}^{N}\left|\sum_{k=\tau+1}^{n}\left(\frac{P_{n-k}}{P_{n}}-\frac{P_{n-k-1}}{P_{n-1}}\right) \lambda_{k}^{(1)} \lambda_{k}^{(2)} \frac{\exp (i k t)}{k+1}\right| .
$$

Then it is enough to prove that

$$
I_{3}^{*}=o\left(\lambda^{(2)}(C / t)\right) \text { as } N \rightarrow \infty \text {. }
$$

Now, we observe that 


$$
\begin{aligned}
& I_{3}^{*} \leq \sum_{n=2 \tau+2}^{N}\left|\sum_{k=\tau+1}^{m}\left(\frac{P_{n-k}}{P_{n}}-\frac{P_{n-k-1}}{P_{n-1}}\right) \lambda_{k}^{(1)} \lambda_{k}^{(2)} \frac{\exp (i k t)}{k+1}\right| \\
& +\sum_{n=2 \tau+2}^{N} \frac{1}{P_{n-1}}\left|\sum_{k=m+1}^{n} p_{n-k} \lambda_{k}^{(1)} \lambda_{k}^{(2)} \frac{\exp (i k t)}{k+1}\right| \\
& +\sum_{n=2 \tau+2}^{N} \frac{p_{n}}{P_{n} P_{n-1}}\left|\sum_{k=m+1}^{n} p_{n-k} \lambda_{k}^{(1)} \lambda_{k}^{(2)} \frac{\exp (i k t)}{k+1}\right| \\
& =I_{31}^{*}+I_{32}^{*}+K_{33}^{*}, \\
& \text { say, where } m=[n / 2] \text {. } \\
& \text { Since }\left\{P_{n-k} / P_{n}\right\} \text { is non-decreasing for each fixed } k \geq 0 \text { and } \\
& |1-\exp (i t)|^{-1}=O\left(t^{-1}\right) \text {, we obtain by an application of Lemma } 1 \text {, } \\
& I_{31}^{*}=\sum_{n=2 \tau+2}^{N}\left|\sum_{k=\tau+1}^{m}\left(\frac{n-k}{P_{n}}-\frac{P_{n-k-1}}{P_{n-1}}\right) \lambda_{k}^{(1)} \lambda_{k}^{(2)} \frac{\exp (i k t)}{k+1}\right| \\
& \leq A \tau \sum_{n=2 \tau+2}^{N}\left(\frac{P_{n-\tau-1}}{P_{n}}-\frac{P_{n-\tau-2}}{P_{n-1}}\right) \lambda_{\tau+1}^{(1)} \lambda_{\tau+2}^{(2)} \frac{|\exp (i(\tau+1) t)|}{\tau+2} \\
& +A \tau \sum_{n=2 \tau+2}^{N}\left(\frac{P_{n-m}}{P_{n}}-\frac{P_{n-m-1}}{P_{n-1}}\right) \lambda_{m}^{(1)} \lambda_{m}^{(2)} \frac{|\exp (i(m+1) t)|}{m+1} \\
& +A \tau \sum_{n=2 \tau+2}^{N}\left|\sum_{k=\tau+1}^{m-1} \Delta\left(\frac{\lambda_{k}^{(1)} \lambda_{k}^{(2)}}{k+1}\right)\left(\frac{n-k}{P_{n}}-\frac{P_{n-k-1}}{P_{n-1}}\right) \exp (i(k+1) t)\right| \\
& +A \tau \sum_{n=2 \tau+2}^{N}\left|\sum_{k=\tau+1}^{m-1}\left(\frac{p_{n-k}}{P_{n}}-\frac{p_{n-k-1}}{P_{n-1}}\right) \frac{\lambda_{k+1}^{(1)} \lambda_{k+1}^{(2)}}{k+2} \exp (i(k+1) t)\right| \text {. }
\end{aligned}
$$

Since for each fixed $k,\left\{p_{n-k} / P_{n}\right\}$ is non-increasing while $\left\{P_{n-k} / P_{n}\right\}$ is non-decreasing, we obtain 


$$
\begin{aligned}
& I_{31}^{*} \leq A \lambda_{\tau+1}^{(1)} \lambda_{\tau+1}^{(2)}\left(\frac{P_{N-\tau-1}}{P_{N}}-\frac{P_{\tau}}{P_{2 \tau+1}}\right)+A \tau \sum_{n=2 \tau+2}^{N}\left(\frac{{ }_{n} P_{n-m^{-}} P_{n}{ }^{P} n-m}{P_{n}{ }^{2} n-1}\right) \frac{\lambda_{m}^{(1)} \lambda_{m}^{(2)}}{m+1} \\
& +A \tau \sum_{k=\tau+1}^{[N / 2]-1} \Delta\left(\frac{\lambda_{k}^{(1)} \lambda_{k}^{(2)}}{k+1}\right) \sum_{n=2 k+1}^{N}\left(\frac{n-k}{P_{n}}-\frac{P_{n-k-1}}{P_{n-1}}\right) \\
& +A \tau \sum_{k=\tau+1}^{[N / 2]-1} \frac{\lambda_{k+1}^{(1)} \lambda_{k+1}^{(2)}}{k+2} \sum_{n=2 k+1}^{N}\left(\frac{P_{n-k-1}}{P_{n-1}}-\frac{P_{n-k}}{P_{n}}\right) \\
& \leq A \lambda^{(2)}(C / t)+A \tau \sum_{n=2 \tau+2}^{N}\left(\frac{p_{m}}{P_{m}}\right) \frac{\lambda_{m}^{(1)} \lambda_{m}^{(2)}}{m+1} \\
& +A \tau \sum_{k=\tau+1}^{[N / 2]-1} \Delta\left(\frac{\lambda_{k}^{(1)} \lambda_{k}^{(2)}}{k+1}\right)+A \tau \sum_{k=\tau+1}^{N} \frac{\lambda_{k+1}^{(1)} \lambda_{k+1}^{(2)}}{k+1} \frac{p_{k}}{P_{2 k}} \\
& \leq A \lambda^{(2)}(c / t)+A \tau p_{\tau+1} \sum_{n=\tau+1}^{N} \frac{\lambda_{n}^{(1)} \lambda_{n}^{(2)}}{n P_{n}} \\
& +A \tau \frac{\lambda_{\tau+1}^{(1)} \lambda(2)}{\tau+1}+A \tau p_{\tau+1} \sum_{k=\tau+1}^{N} \frac{\lambda_{k}^{(1)} \lambda_{k}^{(2)}}{k P_{k}} \\
& \leq A \lambda^{(2)}(C / t)+A \tau p_{\tau+1} \frac{\lambda_{\tau+1}^{(2)}}{P_{\tau+1}}+A \lambda_{\tau+1}^{(2)}+A \tau p_{\tau+1} \frac{\lambda_{\tau+1}^{(2)}}{P_{\tau+1}} \\
& \leq A \lambda^{(2)}(c / t)
\end{aligned}
$$

as $N \rightarrow \infty$, by virtue of the hypotheses (2.6) and that $\left\{\lambda_{k}^{(1)} \lambda_{k}^{(2)} /(k+1)\right\}$ is non-increasing.

$$
\text { Since }\left\{\lambda_{k}^{(1)} \lambda_{k}^{(2)} /(k+1)\right\} \text { is non-increasing, we have by Abel's Lemma }
$$
and Lemma 2, 


$$
\begin{aligned}
I_{32}^{*} & =\sum_{n=2 \tau+2}^{N} \frac{1}{P_{n-1}}\left|\sum_{k=m+1}^{n} p_{n-k} \frac{\lambda_{k}^{(1)} \lambda_{k}^{(2)}}{k+1} \exp (i k t)\right| \\
& \leq A \sum_{n=2 \tau+2}^{N} \frac{1}{P_{n-1}} \frac{\lambda_{m+1}^{(1)} \lambda_{m+1}^{(2)}}{m+2} \max _{m<i \leq n}\left|\sum_{k=m+1}^{2} p_{n-k} \exp (i k t)\right| \\
& \leq A P_{\tau} \sum_{n=\tau}^{N} \frac{\lambda_{n}^{(1)} \lambda_{n}^{(2)} \leq A P_{\tau}}{n P_{n}} \cdot \frac{\lambda_{\tau}^{(2)}}{P_{\tau}} \\
& =O\left(\lambda^{(2)}(c / t)\right)
\end{aligned}
$$

as $N \rightarrow \infty$, by virtue of the hypothesis (2.6).

By a similar method, we have

$$
\begin{aligned}
I_{33}^{*} & =\sum_{n=2 \tau+2}^{N} \frac{p_{n}}{P_{n}^{P}{ }_{n-1}} \sum_{k=m+1}^{n} P_{n-k} \frac{\lambda_{k}^{(1)} \lambda_{k}^{(2)}}{k+1} \exp (i k t) \\
& =\left.\sum_{n=2 \tau+2}^{N} \frac{p_{n}}{P_{n} P_{n-1}}\right|_{k=m+1} ^{n} P_{n-k} \frac{1}{p_{n-k}} p_{n-k} \frac{\lambda_{k}^{(1)} \lambda_{k}^{(2)}}{k+1} \exp (i k t) \mid \\
& \leq A \sum_{n=2 \tau+2}^{N} \frac{p_{n}}{P_{n}{ }^{P} n-1} \frac{P_{m}}{P_{n-m}} \frac{\lambda_{m}^{(1)} \lambda_{m}^{(2)}}{m} \max _{m<i \leq n}\left|\sum_{k=m+1}^{Z} p_{n-k} \exp (i k t)\right| \\
& \leq A P_{\tau} \sum_{n=\tau+1}^{N} \frac{\lambda_{n}^{(1)} \lambda_{n}^{(2)}}{n P_{n}} \leq A P_{\tau} \cdot \frac{\lambda_{\tau}^{(2)}}{P_{\tau}} \\
& =O\left(\lambda^{(2)}(C / t)\right) .
\end{aligned}
$$

Collecting the above estimations $I_{31}^{*}, I_{32}^{*}$, and $I_{33}^{*}$, we prove that uniformly in $0<t \leq \pi, I_{3}^{*}=O\left(\lambda^{(2)}(C / t)\right)$ and a fortiori that $I_{3}=O\left(\lambda^{(2)}(C / t)\right)$. Therefore, by $I_{1}, I_{2}$, and $I_{3}$, we have

$$
I=O\left(\lambda^{(2)}(C / t)\right) \text {. }
$$

This completes the proof of the theorem.

5.

In this section, we consider some applications of our theorem. Using a result of Das and Srivastava (cf. [4], Theorem B), it is shown 
by Kanno [4] that it is possible to deduce the following four corollaries from his theorem. However, if we apply our theorem, we need not appeal to the result of Das and Srivastava (cf. [4], Theorem B) for proofs of their corollaries.

COROLLARY 1 [6]. If

$$
\int_{0}^{\pi} t^{-\alpha}|d \varphi(t)|<\infty
$$

then the series $\sum_{n=1}^{\infty} n^{\alpha} A_{n}(t)$ is sumable $|C, \beta|$ at $t=x$, where $0 \leq \alpha<\beta<1$.

COROLLARY 2. If $0<\alpha<1, \beta \geq 0$, and

$$
\int_{0}^{\pi}(\log C / t)^{\beta}|d \varphi(t)|<\infty \text {, }
$$

then the series $\sum_{n=1}^{\infty}(\log n)^{\beta} A_{n}(t)$ is summable $|c, \alpha|$ at $t=x$.

This corollary coincides to Bosanquet [1] for $\beta=0$, and Mohanty [7] for $B=1$, respectively.

COROLLARY 3. If $1>\alpha \geq 0, \quad \beta \geq 0, \alpha+\beta<1$, and

$$
\int_{0}^{\pi}\left(\log \frac{c}{t}\right)^{\beta}|d \varphi(t)|<\infty \text {, }
$$

then the series

$$
\begin{aligned}
& \sum_{n=0}^{\infty} \frac{A_{n}(t)}{\{\log (n+2)\}^{1-\beta}} \text { is summable }\left|N, 1 /(n+2)\{\log (n+2)\}^{\alpha}\right| . \\
& \text { For } \alpha=\beta=0 \text {, this corollary is due to Varshney [8]. } \\
& \text { COROLLARY 4. If } \\
& \qquad \int_{0}^{\pi}\left(\log \log \frac{C}{t}\right)^{\beta}|d \varphi(t)|<\infty \text { for } 0 \leq \beta<1,
\end{aligned}
$$

then the series 
$\sum_{n=0}^{\infty} \frac{A_{n}(t)}{\log (n+2)\{\log \log (n+2)\}^{1-\beta}}$ is summable $|N, 1 /(n+2) \log (n+2)|$

at $t=x$.

As these corollaries are analogously proved, we shall prove here only Corollary 4.

Proof of Corollary 4. In our theorem, we put $p_{k}=1 /(k+2) \log (k+2)$, $\lambda_{k}^{(1)}=1 / \log (k+2) \log \log (k+2), \lambda_{k}^{(2)}=\{\log \log (k+2)\}^{\beta}$. Then

$$
P_{n}=\sum_{k=0}^{n} 1 /(k+2) \log (k+2) \simeq \log \log (n+2)
$$

Moreover it is easy to see that

$$
\sum_{k=n}^{\infty} \frac{\lambda_{k}^{(1)} \lambda_{k}^{(2)}}{k P_{k}}=o\left(\frac{\{\log \log (n+2)\}^{\beta}}{\log \log (n+2)}\right)=o\left(\frac{\lambda_{n}^{(2)}}{P_{n}}\right) \text {. }
$$

Hence all assumptions of our theorem hold. Therefore the proof is complete. Further, by our theorem, we obtain the following Corollaries 5 and 6 , which correspond to Corollaries 3 and 4 for $\beta=1$, respectively.

COROLLARY 5. If

$$
\int_{0}^{\pi} \log \left(\frac{C}{t}\right)|d \varphi(t)|<\infty
$$

then the series

$$
\sum_{n=0}^{\infty} A_{n}(t) \text { is summable }|N, \log (n+2) /(n+2)| \text {, }
$$

at $t=x$.

Proof. Putting

$$
p_{k}=\log (k+2) /(k+2), \quad \lambda_{k}^{(1)}=1 / \log (k+2), \quad \lambda_{k}^{(2)}=\log (k+2),
$$

then we have

$$
P_{n}=\sum_{k=0}^{n} \frac{\log (k+2)}{k+2} \simeq\{\log (n+2)\}^{2}
$$


On the other hand, we have

$$
\sum_{k=n}^{\infty} \frac{1}{k P_{k}}=o\left(\frac{\log (n+2)}{\{\log (n+2)\}^{2}}\right)=o\left(\frac{\lambda^{(2)}}{P_{n}}\right) .
$$

Therefore, by our theorem, we see that Corollary 5 holds.

By a similar method, we can prove the following corollary.

COROLLARY 6. If

$$
\int_{0}^{\pi}\left\{\log \log \frac{C}{t}\right)|d \varphi(t)|<\infty,
$$

then the series

$$
\begin{aligned}
& \sum_{n=0}^{\infty} A_{n}(t) / \log (n+2) \text { is summable }|N, \log \log (n+2) /(n+2) \log (n+2)|, \\
& \text { at } t=x .
\end{aligned}
$$

\section{References}

[1] L.S. Bosanquet, "The absolute Cesàro summability of a Fourier series", Proc. London Math. Soc. (2) 41 (1936), 517-528.

[2] H.P. DIkshit, "Absolute summability of a Fourier series with factors", preprint.

[3] Masako Izumi and Shin-ichi Izumi, "Absolute Nörlund summability factor of Fourier series", Proc. Japan Acad. 46 (1970), 642-646.

[4] Kōsi Kanno, "On the absolute Nörlund summability of the factored Fourier series", Tôhoku Math. J. 21 (1969), 434-447.

[5] Leonard McFadden, "Absolute Nörlund summability", Duke Math. J. 9 (1942), 168-207.

[6] R. Mohanty, "The absolute Cesàro summability of some series associated with a Fourier series and its allied series", J. London Math. Soc. 25 (1950), 63-67.

[7] R. Mohanty, "Absolute Cesàro summability of a series associated with a Fourier series", Bulz. Calcutta Math. Soo. 44 (1952), 152-154. 
[8] 0.P. Varshney, "On the absolute harmonic summability of a series related to a Fourier series", Proc. Amer. Math. Soc. 10 (1959), $784-789$.

Department of Technology,

Shinshu University,

Nagano,

Japan. 